\title{
Hemostatic Factors and Subclinical Brain Infarction in a Community-Based Sample: The ARIC Study
}

\author{
R.F. Gottesman ${ }^{a} \quad$ C. Cummiskey ${ }^{b} \quad$ L. Chambless ${ }^{\text {c }} \quad$ K.K. Wu ${ }^{g} \quad$ N. Aleksic ${ }^{d}$ \\ A.R. Folsom ${ }^{e}$ A.R. Sharrett ${ }^{f}$

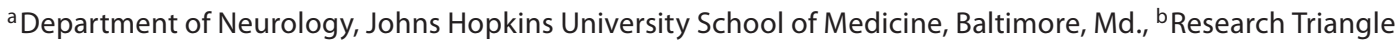 \\ Institute International, ' Department of Biostatistics, University of North Carolina, Chapel Hill, N.C., dUniversity \\ of Texas Health Science Center at Houston, Houston, Tex., e'Division of Epidemiology and Community Health, \\ University of Minnesota School of Public Health, Minneapolis, Minn., and ${ }^{\mathrm{f} D e p a r t m e n t ~ o f ~ E p i d e m i o l o g y, ~ J o h n s ~}$ \\ Hopkins Bloomberg School of Public Health, Baltimore, Md., USA; ${ }^{9}$ National Health Research Institutes, Taiwan, ROC
}

\section{Key Words}

Cerebral infarction - Epidemiology - Magnetic resonance imaging $\cdot$ Hemostatic disorders $\cdot$ Lacunar infarction

\begin{abstract}
Background: Previous data are conflicting as to whether imbalance between hemostatic factors is associated with clinical strokes. We evaluated the association between hemostatic factor levels and subclinical lacunar infarcts in a nested sample from a subset of the Atherosclerosis Risk in Communities (ARIC) cohort. Methods: 196 cases without clinical strokes had lacunar infarcts by MRI, and 214 controls without radiographic infarcts were frequency-matched by age group and sex. Logistic regression models were fitted to assess the association between levels of hemostatic markers and case status. Results: In age-, race- and sex-adjusted models, von Willebrand factor (VWF) and D-dimer were positively associated with case status, with odds ratios for the highest vs. lowest tertile of $2.0(95 \% \mathrm{Cl} 1.2-3.6)$ for vWF and $1.76(95 \% \mathrm{Cl} 1.02-3.0)$ for D-dimer. Plasminogen had nonsignificant inverse associations with presence of silent lacunar infarcts. Conclusions: vWF and D-dimer were positively as-
\end{abstract}

sociated, and plasminogen was nonsignificantly inversely associated with subclinical radiographic infarct. Further studies on the role of these hemostatic factors in the development of silent lacunar infarcts may help elucidate the mechanisms behind this injury and may even point to potential targets for future intervention.

Copyright $\odot 2009$ S. Karger AG, Basel

Hemostatic mechanisms are rarely considered in most stroke patients, despite the key role of hemostatic factors in thrombus formation. Hypercoagulable states may be assessed in young patients with cryptogenic stroke, or hyperviscosity may be considered in individuals with polycythemia vera and ischemic stroke. Hemostatic factors play different roles in thrombus formation and breakdown, and the balance between these factors is likely to be critical in the etiology of diseases related to thrombosis or embolism. Von Willebrand factor (vWF), an endothelial cell product and marker of endothelial cell function, is a key factor in arterial thrombosis, mediating platelet adhesion to sites of vascular injury. $\beta$-Thromboglobulin $(\beta-\mathrm{TG})$ is a specific marker of platelet activation.

\section{KARGER}

(C) 2009 S. Karger AG, Basel

Fax +4161306 1234 E-Mail karger@karger.ch www.karger.com
Accessible online at: www.karger.com/ced
Rebecca F. Gottesman, MD, PhD

Meyer 6-113, 600 North Wolfe Street

Baltimore, MD 21287 (USA)

Tel. +1 410614 2381, Fax +1 4109550672

E-Mail rgottesm@jhmi.edu 
Factors involved in the endogenous fibrinolytic process are also critical in determining likelihood of thrombus formation. D-dimer, a marker of active fibrinolysis, may consequently also be an index of elevated levels of deposited fibrin. Low or impaired fibrinolysis may allow for progression of thrombus.

The Atherosclerosis Risk in Communities (ARIC) study is a biethnic prospective cohort of 15,792 individuals from four centers. In ARIC, fibrinogen, factor VIIIc, vWF, and white blood cell count were positively associated with incident ischemic stroke after adjustment for established vascular risk factors. The adjusted relative risk for vWF in the highest quartile compared to the lowest quartile was 1.71 (95\% CI 1.1-2.7) [1]. Fibrinogen and vWF levels were positively associated with asymptomatic carotid disease [2], as were plasminogen activator inhibitor-1 (PAI-1) antigen, tissue plasminogen activator (tPA) antigen, and D-dimer levels [3].vWF and fibrinogen were positively associated with MRI-detected infarcts [4].

Fisher $[5,6]$ described the histology of lacunar strokes as due to lipohyalinosis and thrombosis of the penetrating branches of the cerebral arteries. Thus, if these small vessels are more prone to occlusion by thrombosis than larger vessels, then any factors that increase the likelihood of thrombosis might preferentially increase the risk of small strokes. The purpose of the current study was to determine if hemostatic and related markers from a more extensive battery added to prediction of subclinical lacunar infarction in a nested sample of the ARIC cohort.

\section{Methods}

\section{Study Population}

The ARIC cohort includes 15,792 individuals who were 45-64 years of age at a baseline examination in 1987-1989. These individuals reside in Forsyth County, N.C., Jackson, Miss. (AfricanAmericans only), Minneapolis, Minn., or Washington County, Md., USA. A subgroup of this larger cohort received research MRI scans, and a further subset of that group, without history of clinical stroke, comprised the cases and controls for this analysis. The ARIC Study has been approved by all participating institutional review boards.

For this particular study, only those persons who also underwent brain MRI at the third ARIC examination in 1993-1995 were included for analysis. At that time 2,825 persons, $\geq 56$ years of age, from Forsyth County or Jackson were invited for MRI. 2\% of women and $6 \%$ of men had contraindications for MRI, $28 \%$ of participants refused the examination, and 1,949 individuals were imaged. Images were of sufficient quality for grading of 1,920 examinations.

Among those who had research MRI scans, a total of 237 infarcts (cases; infarct definition described below) were found, and
Table 1. Exclusions of cases with MRI-detected infarcts and matched controls, ARIC participants aged $\geq 56$ years with cerebral MRI in 1993-1995

$$
\text { Cases Controls }
$$$$
(\mathrm{n}=237) \quad(\mathrm{n}=227)
$$

\begin{tabular}{lrc}
\hline Missing hemostasis values & 13 & 5 \\
Used anticoagulation at visit 1 (1987-89) & 7 & 1 \\
Used anticoagulation at visit 3 (1993-95) & 6 & 1 \\
Had clinical stroke prior to MRI & 15 & 6 \\
\hline Total included & 196 & 214 \\
\hline
\end{tabular}

for comparison a subset (controls, $\mathrm{n}=227$ ) of those with MRI data and not meeting the case definition (not having radiographic infarcts) were selected with frequency matching by sex and age group. After excluding persons with missing hemostasis values, persons known to have taken anticoagulation medications at the baseline examination or at the third ARIC examination, or who had been diagnosed with clinical stroke prior to the MRI (15 cases and 6 controls), 196 cases, with subclinical infarcts, and 214 controls, similar but without subclinical infarcts, remained for analysis (table 1). Because we excluded both cases and controls with clinical stroke, the infarcts identified on the cases' MRI scans were believed to be consistent with subclinical infarction.

MRI and Determination of Stroke Type

The MR scanning protocol has been described in detail [7]. Participants were scanned on 1.5-tesla scanners. Infarct rating was assigned by trained readers at the ARIC MR Reading Center at Johns Hopkins Medical Institutions in Baltimore. Primary image reviewers were board-certified radiologists with subspecialty training in neuroradiology who were blinded to any clinical or demographic information. Infarcts were defined as abnormal signal intensity in a vascular distribution without mass effect. Infarcts were hyperintense to gray matter on both spin density and $\mathrm{T}_{2}$-weighted images, and lesions in white matter had to also be hypointense on $\mathrm{T}_{1}$-weighted images, with intensities similar to cerebrospinal fluid. Using previously established criteria, we analyzed lacunar infarcts, which were defined as being between $3 \mathrm{~mm}$ (smaller abnormalities could not be reliably detected) and $20 \mathrm{~mm}$ in maximum diameter $[8,9]$.

\section{Measurement of Hemostatic Factors}

Blood was drawn using a standardized protocol at the third ARIC visit (1993-1995). Plasma was separated at $4{ }^{\circ} \mathrm{C}$ and stored at $-70^{\circ} \mathrm{C}$ until assayed for hemostatic factors. Methods used by the ARIC hemostasis laboratory for D-dimer, tPA antigen, plasminogen, PAI-1 antigen [10], fibrinogen and vWF [1], soluble thrombomodulin [11], C-reactive protein [12], and $\beta$-TG [13] assays have been described. Reliability coefficients (based on repeated measurements in samples from individuals taken over a few weeks) were 0.83 and 0.81 for $\beta$-TG and PA, respectively; Ddimer, PAI-1, and fibrinogen were intermediate at $0.73,0.72$, and 0.72 , respectively $[14,15]$, and reliability was 0.70 for plasminogen and 0.68 for vWF $[10,16]$. For soluble thrombomodulin, the Pear- 
Table 2. Vascular risk factors in cases with MRI-detected infarcts and matched controls

\begin{tabular}{lccc}
\hline & Cases $(\mathrm{n}=196)$ & Controls $(\mathrm{n}=214)$ & p value $^{*}$ \\
\hline Age, years & 64.4 & 62.5 & (matched) \\
Sex, \% male & 38.8 & 40.2 & $<0.001$ \\
Race, \% black & 62.8 & 43.8 & $<0.001$ \\
Systolic blood pressure, mm Hg & 135 & 125 & $<0.001$ \\
On antihypertensives, \% & 57.8 & 31.9 & 0.003 \\
Current smoking, \% & 27.2 & 14.3 & 0.449 \\
Prevalent CHD, \% at visit 3 & 7.3 & 5.4 & 0.130 \\
Diabetes, \%, fasting glucose $>$ 126 mg/dl & 23.2 & 16.8 & 0.497 \\
LVH by ECG, \% & 5.3 & 3.9 & 0.285 \\
Self-reported aspirin use in past 4 weeks, \% & 49.5 & 44.0 & 0.416 \\
Statin use in past 2 weeks, \% & 4.1 & 5.9 & \\
\hline
\end{tabular}

* For categorical variables, weighted unadjusted proportions are presented. For continuous variables, weighted unadjusted means are presented. $\mathrm{p}$ values are calculated in SUDAAN respecting the stratified random sample design.

son correlation coefficient between the duplicate samples from the same participant was 0.85 ( $n=64$ pairs), the within-pair SD was 14.83 , and the within-pair coefficient of variation was 0.314 [17]. The Pearson correlation coefficient for C-reactive protein was $0.96[12]$.

\section{Data Analysis}

SAS 9.1 was used for primary analyses, and SUDAAN 9.0 was used for the weighted analyses. The sample is a stratified random sample of the ARIC cohort, with one stratum for cases with sampling fraction equal to 1.0, and four strata of controls, based on sex and age group, with varying sampling fractions. Sampling weights were the inverse of the sampling fractions. For descriptive statistics, including tertiles of the hemostasis variable distributions, sampling weights were applied and statistical tests apply methods appropriate to stratified random samples. Unweighted logistic regression models were fit, accounting for the sampling by always adjusting for age, race, and sex, and subsequently with further adjustment for stroke risk factors (current smoker, diabetes, left ventricular hypertrophy (LVH) by electrocardiogram, previous myocardial infarction, coronary artery disease, use of antihypertensive medications, and systolic blood pressure at visit $3)$. Because of recognized differences in levels of some hemostatic factors in blacks versus whites [2], analyses were repeated posthoc separately by race.

\section{Results}

The study includes 196 cases with silent MRI infarcts and 214 age- and sex-matched controls. Cases had higher systolic blood pressure and were more likely to be black, to be smokers, and to take antihypertensive medications (table 2). The weighted median, lower, and upper tertile values for each of the hemostatic variables tested are displayed in table 3. Weighted median (and mean) levels of vWF, fibrinogen, $D$-dimer, and $\beta$-TG were higher in cases than controls.

In models including only one hemostatic variable at a time (not shown), the odds ratios (OR) for case status for vWF levels in the highest vs. the lowest tertile were 2.04, 95\% CI 1.18-3.55 after age, race and sex adjustment and $1.74,95 \%$ CI $0.96-3.15$ in fully adjusted models (covariates as listed in table 4). Similar analysis showed D-dimer associated with case status (age/sex/race-adjusted OR 1.76, 95\% CI 1.02, 3.02; fully adjusted OR 1.48, 95\% CI $0.82,2.67)$. Plasminogen had an inverse although nonsignificant and lacking dose-response association in both models (demographic-adjusted OR 0.78, 95\% CI 0.46, 1.32 ; fully adjusted OR $0.69,95 \%$ CI $0.39,1.22$, comparing highest tertile to lowest tertile).

These relationships persisted in models including two hemostatic variables together, demonstrating positive associations with higher values of vWF and D-dimer and inverse associations with higher levels of plasminogen. The associations with vWF and D-dimer were stronger after accounting for plasminogen. These results are displayed in table 4, including models with both demographic adjustments and demographic plus risk factor adjustments.

Although univariate associations were significant for several hemostatic variables (table 3), neither fibrinogen nor $\beta$-TG remained significant in adjusted models. However, the addition of the combination of vWF and plas- 
Table 3. Weighted medians and tertiles of the hemostasis variables, and p values for tests for differences between cases and controls for medians

\begin{tabular}{llll}
\hline Hemostatic factor & $\begin{array}{l}\text { Cases, median (lower, } \\
\text { upper tertile cut points) }\end{array}$ & $\begin{array}{l}\text { Controls, median (lower, } \\
\text { upper tertile cut points) }\end{array}$ & p value \\
\hline vWF, \% & $148(130,169)$ & $133(107,147)$ & 0.01 \\
Fibrinogen, mg/dl & $360(326,389)$ & $343(313,379)$ & 0.05 \\
Plasminogen, \% & $111(105,120)$ & $113(107,119)$ & 0.63 \\
D-dimer, ng/ml & $449(365,555)$ & $378(278,448)$ & 0.004 \\
$\beta$-TG, ng/ml & $18.6(14.4,24.0)$ & $15.4(13.8,18.5)$ & 0.002 \\
TPA antigen, ng/ml & $9.96(7.96,13.11)$ & $9.11(7.46,11.66)$ & 0.16 \\
PAI-1 antigen, ng/ml & $16.4(10.4,25.0)$ & $16.2(10.6,25.0)$ & 0.90 \\
C-reactive protein, mg/dl & $3.17(1.84,5.51)$ & $2.55(1.20,4.54)$ & 0.27 \\
Soluble thrombomodulin, $\mathrm{ng} / \mathrm{ml}$ & $27.2(23.2,34.7)$ & $29.4(22.9,36.2)$ & 0.25 \\
\hline
\end{tabular}

Table 4. Adjusted ORs of 2 nd and 3rd tertile values of hemostatic factors (vWF, plasminogen, D-dimer) for radiographically present cerebral infarcts (with 95\% CIs), in models with combined pairs of hemostatic factors in addition to other variables for adjustment

\begin{tabular}{lllll}
\hline & Model A* & Model B** & Model C* & Model D** $^{* *}$ \\
\hline vWF & & & \\
2nd vs. 1st tertile & $1.4(0.8,2.5)$ & $1.4(0.8,2.6)$ & \\
3rd vs. 1st tertile & $2.2(1.2,3.8)$ & $1.9(1.0,3.4)$ & & \\
Plasminogen & & & & \\
2nd vs. 1st tertile & $0.5(0.3,0.8)$ & $0.4(0.2,0.8)$ & $0.5(0.3,0.8)$ & $0.4(0.2,0.7)$ \\
3rd vs. 1st tertile & $0.7(0.4,1.2)$ & $0.6(0.4,1.2)$ & $0.7(0.4,1.2)$ & $0.6(0.4,1.2)$ \\
D-dimer & & & $1.4(0.8,2.3)$ & $1.1(0.6,2.1)$ \\
2nd vs. 1st tertile & & & $1.9(1.1,3.3)$ & $1.6(0.9,2.9)$ \\
3rd vs. 1st tertile & & & & \\
\hline
\end{tabular}

* Adjusted for age, race, and sex.

** Adjusted for age, race, sex, current smoking, diabetes, LVH by ECG, previous MI, coronary artery disease, use of antihypertensives, systolic blood pressure.

minogen to the fully adjusted model with risk factors (table 4 , model B) was significant ( -2 log likelihood difference $=12.23\left(\chi^{2}\right), 4$ d.f., $\left.\mathrm{p}=0.016\right)$. The addition of plasminogen and $\mathrm{D}$-dimer to the adjusted model with risk factors (table 4 , model D) also added significantly to the model ( $\chi^{2}=16.57,4$ d.f., $\left.\mathrm{p}=0.002\right)$.

Similar associations were observed in blacks and whites studied separately (not shown in tables), although point estimates for vWF in the models with plasminogen were generally higher in blacks, with decreased power due to smaller sample sizes in these separate groups (model A, 2nd (middle) vs. 1st (lowest) tertile, OR 2.17 $(0.93,5.08), 3 \mathrm{rd}$ (highest) vs. 1st (lowest) tertile, 2.61 (1.18, $5.75))$ than in whites (2nd vs. 1st tertile, OR $1.05(0.49$,
2.27), 3rd vs. 1st tertile: $2.25(0.98,5.20))$. For model B, point estimates for the association between $\mathrm{vWF}$ and case status were higher for blacks in both tertile comparisons than for whites. Mean (and median) levels of vWF, D-dimer and plasminogen were all higher in blacks than in whites, for both cases and controls.

\section{Conclusions}

vWF and D-dimer were positively associated, and plasminogen was nonsignificantly inversely associated with subclinical radiographic infarct. These results are similar to the associations of vWF previously reported 
with clinical stroke, other MRI abnormalities [4], and of both vWF and D-dimer with peripheral arterial disease [18]. In addition, our analysis includes additional results with a more extensive panel of hemostatic and inflammatory factors. Our findings, in combination with these and other previous observations, emphasize the potential importance of hemostatic abnormalities in the development of both clinical and subclinical vascular disease of the brain; thus, similar preventive strategies may be effective.

The growing body of evidence supporting the importance of factors such as vWF, plasminogen and D-dimer in stroke prediction may point to a potential future target in the prevention of stroke or of subclinical stroke. Several of these markers are not only hemostatic in mechanism, but are potential markers of endothelial dysfunction (vWF) or inflammation (fibrinogen), which may further support a clinically relevant mechanism. In a study in swine, the specific use of a monoclonal antibody to $\mathrm{vWF}$, for example, led to an $81 \%$ reduction in platelet deposition on de-endothelialized vessel wall, when compared with a $30 \%$ reduction from aspirin [19]. More investigation is needed in this area.

Results similar to ours were found in a Japanese group of 123 asymptomatic hypertensive subjects $>55$ years of age, where clinically silent infarcts detected by MRI were associated with elevated levels of PAI-1, D-dimer, and vWF. In that study all subclinical infarcts were lacunar infarcts of $<15 \mathrm{~mm}$ in diameter [20]. This association of hemostatic factors with smaller lacunar infarcts is consistent with pathologic theories of lacunar infarction. Although the purpose of the hemostatic system is to prevent excessive blood loss in the event of injury to a blood vessel, this process can become pathologic and lead to thrombosis and vessel occlusion. This may be particularly concerning in smaller-sized vessels, as the smaller caliber of these vessels may make them more easily blocked by thrombus or emboli. This potential preference of hemostatic factors for lacunar infarction was not supported, however, in one study in which individuals with lacunar versus cortical strokes did not differ in levels of endogenous fibrinolytic factors, PAI-1 and tPA in blood drawn within 7 days following the stroke [21].

Knowledge of hemostatic abnormalities is useful not only for understanding the pathogenesis of small vessel infarction, but potentially also for evaluating overall stroke risk. Recent reports of stroke prediction have concentrated on not only the 'traditional' risk factors but have expanded to include nontraditional risk factors, including hemostatic factors. Hemostatic markers may help in predicting both stroke and outcome among patients with clinical stroke. Elevated levels of vWF and $\beta$-TG were associated with higher mortality among subjects with history of clinical stroke [22]. Many prospective cohort studies have shown increased rates of stroke and other cardiovascular disease in individuals with elevated levels of certain markers of hemostasis. In the Caerphilly cohort of over 2,000 men in Scotland, traditional risk factors were associated with a hazard ratio for cardiovascular disease of 2.7 when top and bottom tertiles of the prediction score were compared, but addition of four hemostatic factors (PAI-1, fibrinogen, D-dimer, and factor VIIc) increased the hazard ratio of the prediction score to 3.7. Three of these hemostatic factors (PAI-1, D-dimer, and thrombin-antithrombin complexes) were also stronger predictors of ischemic stroke (e.g. HR 2.25 for D-dimer) than of coronary heart disease (HR 1.03 for highest levels) [23]. Fibrinogen was both a univariate predictor of ischemic stroke and remained significant after multivariate adjustment in the Edinburgh Artery study, as did tPA and D-dimer [24]. The addition of markers of endothelial cell dysfunction, such as vWF, when added to clinical stroke risk calculation have been noted to improve prediction of risk of subsequent stroke in moderate risk patients [25].

One limitation of our study is the cross-sectional design. It is possible, though perhaps unlikely, given the small size of the silent infarcts studied, that the cerebral infarction itself may have affected our measures of systemic hemostasis, and not vice versa. Given the cross-sectional design, the direction of the relationship cannot be determined. Another limitation of the case-control design is that there may have been other unmeasured confounders, which could be associated both with elevated vWF, for instance (likely reflecting endothelial activation), and with silent cerebral infarction. We did not exclude individuals on antiplatelet medications, since this would have dramatically reduced the sample size, but it is possible that the use of antiplatelet medications could also confound the results. Our sample size was relatively small, with sufficient power to detect only the most moderate associations. Generally, the associations were modest, and in multivariate models many of the associations lost statistical significance. Finally, the fact that hemostatic factors used were measured at a single time point with only moderate reliability may have limited the strength of our study.

Despite these limitations, the findings support a possible role for hemostatic factors in subclinical cerebral ischemia. The study is strengthened, however, by the bi- 
ethnic distribution of its cohort, allowing analysis which showed similar associations by race. This is particularly important because black and white participants have different levels of hemostatic factors. The study is also strengthened by the broad panel of hemostatic factors measured and the standardized definition of radiographic infarct.

Silent brain infarcts were modestly associated in this study with higher levels of vWF and D-dimer and with lower levels of plasminogen. Further studies on the role of these and other hemostatic factors in the development of silent infarcts and white matter ischemic disease may help elucidate the mechanisms behind this injury and may even point to potential targets for future intervention in stroke prevention.

\section{Acknowledgements}

The Atherosclerosis Risk in Communities Study is carried out as a collaborative study supported by the National Heart, Lung, and Blood Institute contracts N01-HC-55015, N01-HC-55016, N01-HC-55018, N01-HC-55019, N01-HC-55020, N01-HC-55021, and N01-HC-55022. The authors thank the staff and participants of the ARIC Study for their important contributions.

\section{References}

-1 Folsom AR, Rosamond WD, Shahar E, Cooper LS, Aleksic N, Nieto FJ, Rasmussen ML, Wu KK: Prospective study of markers of hemostatic function with risk of ischemic stroke. Circulation 1999;100:736-742.

$\checkmark 2$ Folsom AR, Wu KK, Shahar E, Davis CE: Association of hemostatic variables with prevalent cardiovascular disease and asymptomatic carotid artery atherosclerosis. The Atherosclerosis Risk in Communities (ARIC) Study Investigators. Arterioscler Thromb 1993;13:1829-1836.

-3 Salomaa V, Stinson V, Kark JD, Folsom AR, Davis CE, Wu KK: Association of fibrinolytic parameters with early atherosclerosis. The ARIC Study. Circulation 1995;91:284290

-4 Knuiman MW, Folsom AR, Chambless LE, Liao D, Wu KK: Association of hemostatic variables with MRI-detected cerebral abnormalities: The Atherosclerosis Risk in Communities Study. Neuroepidemiology 2001; 20:96-104.

5 Fisher CM: Lacunes: small, deep cerebral infarcts. Neurology 1965;15:774-784.

-6 Fisher CM: Lacunar strokes and infarcts: a review. Neurology 1982;32:871-876

$\checkmark 7$ Knopman DS, Mosley TH, Catellier DJ, Sharrett AR: Cardiovascular risk factors and cerebral atrophy in a middle-aged cohort. Neurology 2005;65:876-881

-8 Longstreth WT Jr, Bernick C, Manolio TA, Bryan N, Jungreis CA, Price TR: Lacunar infarcts defined by magnetic resonance imaging of 3,660 elderly people. Arch Neurol 1998;55:1217-1225.

-9 Longstreth WT Jr, Dulberg C, Manolio TA, Lewis MR, Beauchamp NJ Jr, O'Leary D, Carr J, Furberg CD: Incidence, manifestations, and predictors of brain infarcts defined by serial cranial magnetic resonance imaging in the elderly. The Cardiovascular Health Study. Stroke 2002;33:2376-2382.
10 Folsom AR, Aleksic N, Park E, Salomaa V, Juneja H, Wu KK: Prospective study of fibrinolytic factors and incident coronary heart disease: The Atherosclerosis Risk in Communities (ARIC) Study. Arterioscler Thromb Vasc Biol 2001;21:611-617.

$>11$ Wu KK, Aleksic N, Ballantyne CM, Ahn C, Juneja $\mathrm{H}$, Boerwinkle E: Interaction between soluble thrombomodulin and intracellular adhesion molecule-1 in predicting risk of coronary heart disease. Circulation 2003; 107:1729-1732.

12 Folsom AR, Aleksic N, Catellier D, Juneja HS, Wu KK: C-reactive protein and incident coronary heart disease in the Atherosclerosis Risk in Communities (ARIC) Study. Am Heart J 2002;144:233-238.

13 Ghaddar HB, Cortes J, Salomaa V, Kark JD, Davis CE, Folsom AR, Heiss G, Stinson V, Wu KK: Correlation of specific platelet activation markers with carotid arterial wall thickness. Thromb Haemost 1995;74:943-948.

14 Nguyen ND, Ghaddar H, Stinson V, Chambless LE, Wu KK: ARIC Hemostasis Study IV. Intraindividual variability and reliability of hemostatic factors. The Atherosclerosis Risk in Communities (ARIC). Thromb Haemost 1995;73:256-260.

15 Sturgeon JD, Folsom AR, Longstreth WT Jr, Shahar E, Rosamond WD, Cushman M: Hemostatic and inflammatory risk factors for intracerebral hemorrhage in a pooled cohort. Stroke 2008;39:2268-2273.

16 Chambless LE, McMahon R, Wu K, Folsom AR, Finch A, Shen YL: Short-term intraindividual variability in hemostasis factors: the ARIC Study. Ann Epidemiol 1992;2:723733.

17 Salomaa V, Matei C, Aleksic N, SansoresGarcia L, Folsom AR, Juneja H, Chambless LE, Wu KK: Soluble thrombomodulin as a predictor of incident coronary heart disease and symptomless carotid artery atheroscle- rosis in the Atherosclerosis Risk in Communities (ARIC) Study: a case-cohort study. Lancet 1999;353:1729-1734

18 Reich LM, Heiss G, Boland LL, Hirsch AT, Wu K, Folsom AR: Ankle-brachial index and hemostatic markers in the Atherosclerosis Risk in Communities (ARIC) Study cohort. Vasc Med 2007;12:267-273.

19 Ip JH, Fuster V, Badimon L, Badimon J, Taubman MB, Chesebro JH: Syndromes of accelerated atherosclerosis: role of vascular injury and smooth muscle cell proliferation. J Am Coll Cardiol 1990;15:1667-1687.

-20 Kario K, Matsuo T, Kobayashi H, Hoshide S, Shimada K: Hyperinsulinemia and hemostatic abnormalities are associated with silent lacunar cerebral infarcts in elderly hypertensive subjects. J Am Coll Cardiol 2001; 37:871-877

21 Lindgren A, Lindoff C, Norrving B, Astedt B, Johansson BB: Tissue plasminogen activator and plasminogen activator inhibitor-1 in stroke patients. Stroke 1996;27:1066-1071.

22 Carter AM, Catto AJ, Mansfield MW, Bamford JM, Grant PJ: Predictive variables for mortality after acute ischemic stroke. Stroke 2007;38:1873-1880.

-23 Smith A, Patterson C, Yarnell J, Rumley A, Ben-Schlomo Y, Lowe G: Which hemostatic markers add to the predictive value of conventional risk factors for coronary heart disease and ischemic stroke? The Caerphilly Study. Circulation 2005;112:3080-3087.

-24 Smith FB, Lee AJ, Fowkes FGR, Price JF, Rumley A, Lowe GDO: Hemostatic factors as predictors of ischemic heart disease and stroke in the Edinburgh Artery Study. Arterioscler Thromb Vasc Biol 1997;17:33213325 .

25 Lip GYH, Watson T, Shantsila E: Anticoagulation for stroke prevention in atrial fibrillation: is gender important? Eur Heart J 2006; 27:1893-1894. 